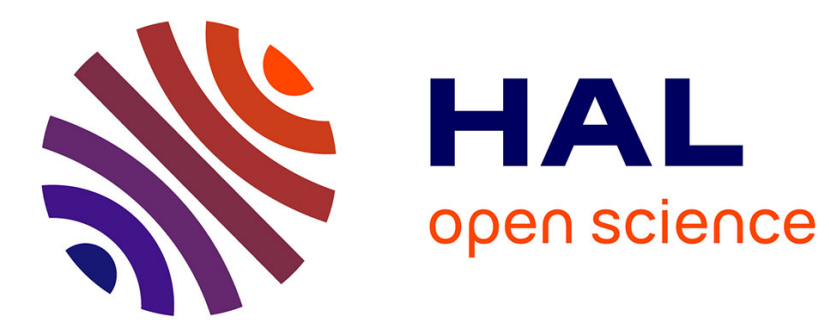

\title{
On adaptive strategies and error control in fracture mechanics
}

\author{
Per Heintz, Klas Samuelsson
}

\section{To cite this version:}

Per Heintz, Klas Samuelsson. On adaptive strategies and error control in fracture mechanics. Computers \& Structures, 2004, 82 (6), pp.485 - 497. 10.1016/j.compstruc.2003.10.013 . hal-01716698

\section{HAL Id: hal-01716698 \\ https://hal.science/hal-01716698}

Submitted on 24 Feb 2018

HAL is a multi-disciplinary open access archive for the deposit and dissemination of scientific research documents, whether they are published or not. The documents may come from teaching and research institutions in France or abroad, or from public or private research centers.
L'archive ouverte pluridisciplinaire HAL, est destinée au dépôt et à la diffusion de documents scientifiques de niveau recherche, publiés ou non, émanant des établissements d'enseignement et de recherche français ou étrangers, des laboratoires publics ou privés. 


\title{
On adaptive strategies and error control in fracture mechanics
}

\author{
Per Heintz ${ }^{\mathrm{a}, *}$, Klas Samuelsson ${ }^{\mathrm{b}}$ \\ a Department of Applied Mechanics, Chalmers University of Technology, SE-412 96 Göteborg, Sweden \\ ${ }^{\mathrm{b}}$ Department of Mathematics, Chalmers University of Technology, SE-412 96 Göteborg, Sweden
}

\begin{abstract}
Finite element approximations in elastic fracture mechanics are traditionally carried out on a priori constructed meshes with singularity elements that surrounds the crack tip. In this contribution we discuss an adaptive algorithm based on goal oriented error measures using ordinary elements and a $p$-refinement for the linearization of the secant forms. The numerical experiments show that good results can be obtained without imposition of hands before the computations starts. Furthermore, we note that it is often possible, for engineering goal quantities, to linearize the data to the dual problem such that additional discretization error is avoided, and the error representation formula is in a sense trivial.
\end{abstract}

Keywords Fracture mechanics; Error control; Adaptivity; Energy release rate

\section{Introduction}

Specifying a goal quantity a priori makes it possible to formulate a corresponding dual problem that describes the error transport in the domain, related to the particular quantity of interest. The dual problem can be approximated numerically and further used in an adaptive algorithm in order to adapt the mesh to the pre-specified goal, i.e., goal oriented adaptivity. A posteriori error estimates based on duality was introduced in the 1990s by Johnson and co-workers [1-3] and have also been used in nonlinear solid mechanics by Rannacher and co-workers in [4,5]. See also Larsson [6] for further developments.

The main difference between the dual-problem methodology and other standard adaptive schemes (such as stress/strain projections) is that it is possible to adapt the grid to a pre-specified goal quantity by solving the corresponding dual problem. The main advantages are that the degrees of freedom are distributed in a more

\footnotetext{
${ }^{*}$ Corresponding author.

E-mail address: per.heintz@me.chalmers.se (P. Heintz).
}

effective manner and that you get an estimation of the error in the goal quantity. However, the cost of solving a global dual problem in an enriched FE space should be considered and compared to the computational cost using traditional methods which are based on postprocessing the solution locally, either on element or element-patch level.

In this contribution we propose an adaptive algorithm based on a global p-refinement of the primal problem. The finer approximation is used in the linearization of the secant form of the error measure. We show that the error representation formula for the error in the goal quantity turns out to be trivial if the dual problem is then approximated in the same $p$-refined function space. We also consider using the results from the finer primal problem.

Prediction of instability of a pre-existing macroscopic crack is often based on the assumption that the material in the vicinity of the crack behaves in a linear elastic fashion, so that the stress field has a singularity at the crack tip. The crack is defined as being unstable when the stress intensity reaches a critical material dependent level, and is traditionally obtained from the FE approximation by studying crack boundary movements 
or using an integral formulation of the energy release rate at crack growth. Although much research has been done in this field there is little attention paid to the analysis of discretization errors in the FE approximation. For instance, the design of the finite element mesh is often made a priori instead of using adaptive techniques. In the numerical experiments we investigate how well the adaptive algorithm resolves approximations encountered in fracture mechanics. We do not use special singularity elements or other special techniques since the purpose is to exploit the nature of the algorithm in a fundamental fashion. For background material on the considered energy release rate methods we refer to [7-9].

The outline of the paper is as follows: In Section 2 we recall the Gateaux derivative and the nonlinear Galerkin orthogonality which are essential to the error analysis on which the adaptive algorithm is based. In Section 3 we derive the error representation formula, based on duality arguments, for an arbitrary choice of goal functional. Furthermore we discuss how the continuous dual problem can be linearized in a computational setting. In Section 4 we describe the adaptive strategy and mention some different mesh refinement options. In Section 5, a model problem is formulated and we present the considered goal quantities used in the simulations. In Section 6 we present our numerical results and finally, in Section 7 , we present some concluding remarks.

\section{Preliminaries}

In the following sections we denote a test function $v$ and a direction of the Gateaux derivative $\boldsymbol{w}$. The $d$ dimensional function space $\boldsymbol{V}$ is defined as

$\boldsymbol{V} \stackrel{\text { def }}{=}\left\{\mathrm{v}_{\mathrm{i}}: \int \mathrm{v}_{\mathrm{i}}^{2}+\left|\nabla \mathrm{v}_{\mathrm{i}}\right|^{2}<\infty, \quad \mathrm{i}=1, \ldots, \mathrm{d}\right\}$,

with corresponding finite element subspace $\boldsymbol{V}_{h}$.

The variational and FE formulation of a linear PDE is described in terms of its bilinear $a(\boldsymbol{u}, \boldsymbol{v})$ and linear form $L(\boldsymbol{v})$; find $\boldsymbol{u} \in \boldsymbol{V}, \boldsymbol{u}_{h} \in \boldsymbol{V}_{h}$ :

$a(\boldsymbol{u}, \boldsymbol{v})=L(\boldsymbol{v}) \quad \forall \boldsymbol{v} \in \boldsymbol{V}$,

$a\left(\boldsymbol{u}_{h}, \boldsymbol{v}\right)=L(\boldsymbol{v}) \quad \forall \boldsymbol{v} \in \boldsymbol{V}_{h}$.

The Galerkin orthogonality for linear operators is obtained subtracting (2) from (1)

$a(\boldsymbol{e}, \boldsymbol{v})=0 \quad \forall \boldsymbol{v} \in \boldsymbol{V}_{h}$,

where $\boldsymbol{e} \stackrel{\text { def }}{=} \boldsymbol{u}-\boldsymbol{u}_{\mathrm{h}}$. For nonlinear operators, the corresponding variational and $\mathrm{FE}$ formulation reads; find $\boldsymbol{u} \in \boldsymbol{V}, \boldsymbol{u}_{h} \in \boldsymbol{V}_{h}$ :

$a(\boldsymbol{u} ; \boldsymbol{v})=L(\boldsymbol{v}) \quad \forall \boldsymbol{v} \in \boldsymbol{V}$,

$a\left(\boldsymbol{u}_{h} ; \boldsymbol{v}\right)=L(\boldsymbol{v}) \quad \forall \boldsymbol{v} \in \boldsymbol{V}_{h}$, where $a(\cdot ; \cdot)$ is the semi-linear form (nonlinear in the test and linear in the trial argument).

The Gateaux derivative of the semi-linear form with respect to its first argument is defined as

$a^{\prime}(\boldsymbol{u} ; \boldsymbol{w}, \boldsymbol{v}) \stackrel{\text { def }}{=} \frac{\partial}{\partial \epsilon} \mathrm{a}(\boldsymbol{u}+\epsilon \boldsymbol{w} ; \boldsymbol{v})_{\mid \epsilon=0}$.

The secant form of $a(\boldsymbol{u} ; \boldsymbol{v})$ is now obtained as

$a_{\mathrm{S}}\left(\boldsymbol{u}, \boldsymbol{u}_{h} ; \boldsymbol{w}, \boldsymbol{v}\right) \stackrel{\text { def }}{=} \int_{0}^{1} \mathrm{a}^{\prime}(\overline{\boldsymbol{u}}(\mathrm{s}) ; \boldsymbol{w}, \boldsymbol{v}) \mathrm{ds}$,

where $\overline{\boldsymbol{u}}(s) \stackrel{\text { def }}{=} \boldsymbol{u}_{\mathrm{h}}+$ se . The choice $\boldsymbol{w}=\boldsymbol{e}$ leads to

$$
\begin{aligned}
a_{\mathrm{S}}\left(\boldsymbol{u}, \boldsymbol{u}_{h} ; \boldsymbol{e}, \boldsymbol{v}\right) & =\int_{0}^{1} a^{\prime}(\overline{\boldsymbol{u}}(s) ; \boldsymbol{e}, \boldsymbol{v}) \mathrm{d} s \\
& =a(\boldsymbol{u} ; \boldsymbol{v})-a\left(\boldsymbol{u}_{h} ; \boldsymbol{v}\right) .
\end{aligned}
$$

The nonlinear Galerkin orthogonality is now formulated using (4) to substitute the first term in (8)

$$
\begin{aligned}
& a_{\mathrm{S}}\left(\boldsymbol{u}, \boldsymbol{u}_{h} ; \boldsymbol{e}, \boldsymbol{v}\right)=L(\boldsymbol{v})-a\left(\boldsymbol{u}_{h} ; \boldsymbol{v}\right) \quad \forall \boldsymbol{v} \in \boldsymbol{V}, \\
& a_{\mathrm{S}}\left(\boldsymbol{u}, \boldsymbol{u}_{h} ; \boldsymbol{e}, \boldsymbol{v}\right)=0 \quad \forall \boldsymbol{v} \in \boldsymbol{V}_{h} .
\end{aligned}
$$

\section{Error analysis based on duality}

\subsection{The error representation formula}

Let $Q(\boldsymbol{u})$ be a quantity of physical interest derived from the solution $\boldsymbol{u}$. To measure the error in the discretization we introduce $E\left(\boldsymbol{u}, \boldsymbol{u}_{h}\right)$ as

$E\left(\boldsymbol{u}, \boldsymbol{u}_{h}\right) \stackrel{\text { def }}{=} \mathrm{Q}(\boldsymbol{u})-\mathrm{Q}\left(\boldsymbol{u}_{\mathrm{h}}\right)$.

To establish the variational format of the dual problem, on which the a posteriori error estimate is based, we use the Gateaux derivative for $E\left(\boldsymbol{u}, \boldsymbol{u}_{h}\right)$ with respect to its first argument

$\left.E^{\prime}\left(\boldsymbol{u}, \boldsymbol{u}_{h} ; \boldsymbol{w}\right) \stackrel{\text { def }}{=} \frac{\partial}{\partial \epsilon} \mathrm{E}\left(\boldsymbol{u}+\epsilon \boldsymbol{w}, \boldsymbol{u}_{\mathrm{h}}\right)\right|_{\epsilon=0}=\nabla \mathrm{Q}(\boldsymbol{u}) \cdot \boldsymbol{w}$.

We now define the secant form of $E\left(\boldsymbol{u}, \boldsymbol{u}_{h}\right)$ as

$$
\begin{aligned}
E_{\mathrm{S}}\left(\boldsymbol{u}, \boldsymbol{u}_{h} ; \boldsymbol{w}\right) & \stackrel{\text { def }}{=} \int_{0}^{1} \mathrm{E}^{\prime}\left(\overline{\boldsymbol{u}}(\mathrm{s}), \boldsymbol{u}_{\mathrm{h}} ; \boldsymbol{w}\right) \mathrm{ds} \\
& =\int_{0}^{1} \nabla \mathrm{Q}(\overline{\boldsymbol{u}}(\mathrm{s})) \cdot \boldsymbol{w} \mathrm{ds},
\end{aligned}
$$

The choice $\boldsymbol{w}=\boldsymbol{e}$ leads to

$E_{\mathrm{S}}\left(\boldsymbol{u}, \boldsymbol{u}_{h} ; \boldsymbol{e}\right)=E\left(\boldsymbol{u}, \boldsymbol{u}_{h}\right)-E\left(\boldsymbol{u}_{h}, \boldsymbol{u}_{h}\right)=E\left(\boldsymbol{u}, \boldsymbol{u}_{h}\right)$.

For given $\boldsymbol{u}$ and $\boldsymbol{u}_{h}$ we recall the secant form of the primal problem (7), and define the corresponding dual bilinear form $a_{\mathrm{S}}^{*}\left(\boldsymbol{u}, \boldsymbol{u}_{h} ; \boldsymbol{w}, \boldsymbol{v}\right)$ such that 
$a_{\mathrm{S}}^{*}\left(\boldsymbol{u}, \boldsymbol{u}_{h} ; \boldsymbol{w}, \boldsymbol{v}\right) \stackrel{\text { def }}{=} \mathrm{a}_{\mathrm{S}}\left(\boldsymbol{u}, \boldsymbol{u}_{\mathrm{h}} ; \boldsymbol{v}, \boldsymbol{w}\right)$.

The variational format of the dual problem is now formulated as; find $\boldsymbol{\varphi} \in \boldsymbol{V}$ :

$a_{\mathrm{S}}^{*}\left(\boldsymbol{u}, \boldsymbol{u}_{h} ; \boldsymbol{\varphi}, \boldsymbol{v}\right)=E_{\mathrm{S}}\left(\boldsymbol{u}, \boldsymbol{u}_{h}, \boldsymbol{v}\right) \quad \forall \boldsymbol{v} \in \boldsymbol{V}$.

Using (15), (16) and (9) the following exact error representation holds where we have chosen $\boldsymbol{v}=\boldsymbol{e}$ :

$$
\begin{aligned}
E_{\mathrm{S}}\left(\boldsymbol{u}, \boldsymbol{u}_{h} ; \boldsymbol{e}\right) & =a_{\mathrm{S}}^{*}\left(\boldsymbol{u}, \boldsymbol{u}_{h} ; \boldsymbol{\varphi}, \boldsymbol{e}\right)=a_{\mathrm{S}}\left(\boldsymbol{u}, \boldsymbol{u}_{h} ; \boldsymbol{e}, \boldsymbol{\varphi}\right) \\
& =a_{\mathrm{S}}\left(\boldsymbol{u} ; \boldsymbol{e}, \boldsymbol{u}_{h}, \boldsymbol{\varphi}-\pi_{h} \boldsymbol{\varphi}\right) \\
& =L\left(\boldsymbol{\varphi}-\pi_{h} \boldsymbol{\varphi}\right)-a\left(\boldsymbol{u}_{h} ; \boldsymbol{\varphi}-\pi_{h} \boldsymbol{\varphi}\right) .
\end{aligned}
$$

The third equality is obtained using the Galerkin orthogonality (10) to insert an interpolant $\pi_{h} \varphi$ that belongs to $\boldsymbol{V}_{h}$.

Using (14) and (11) we obtain

$$
\begin{aligned}
E\left(\boldsymbol{u}, \boldsymbol{u}_{h}\right) & =L\left(\boldsymbol{\varphi}-\pi_{h} \boldsymbol{\varphi}\right)-a\left(\boldsymbol{u}_{h}, \boldsymbol{\varphi}-\pi_{h} \boldsymbol{\varphi}\right) \\
& =\sum_{K \in T_{h}}\left(L\left(\boldsymbol{\varphi}-\pi_{h} \boldsymbol{\varphi}\right)-a\left(\boldsymbol{u}_{h}, \boldsymbol{\varphi}-\pi_{h} \boldsymbol{\varphi}\right)\right)_{K},
\end{aligned}
$$

$\left|E\left(\boldsymbol{u}, \boldsymbol{u}_{h}\right)\right|=\left|\sum_{K \in T_{h}}\left(L\left(\boldsymbol{\varphi}-\pi_{h} \boldsymbol{\varphi}\right)-a\left(\boldsymbol{u}_{h}, \boldsymbol{\varphi}-\pi_{h} \boldsymbol{\varphi}\right)\right)_{K}\right|$,

where the summation is carried out over every element $K$ in the mesh $T_{h}$. Eq. (19) is the exact error representation formula that will be used in the adaptive routine.

Remark. For two chosen function spaces, $\boldsymbol{V}_{h}$ and a larger discrete function space $\boldsymbol{V}_{h^{+}}, E_{\mathrm{S}}\left(\boldsymbol{u}, \boldsymbol{u}_{h} ; \boldsymbol{v}\right)$ can be linearized exactly for goal quantities that are at most quadratic in $\boldsymbol{u}$ (since the gradient is then linear in $\boldsymbol{u}$ ). The error representation formula for bilinear forms, with $\boldsymbol{\varphi} \in \boldsymbol{V}_{h^{+}}$, is thus trivial with respect to the considered function spaces.

\subsection{Discretizing the dual problem}

Solving the dual problem (16) first requires a linearization of the functional $E_{\mathrm{S}}\left(\boldsymbol{u}, \boldsymbol{u}_{h} ; \boldsymbol{v}\right)$. Nonlinear error measures can be linearized using the rectangle (20), trapezoidal (21) or midpoint (22) rule.

$$
\begin{aligned}
& \int_{0}^{1} \nabla Q(\overline{\boldsymbol{u}}(s)) \cdot \boldsymbol{w} \mathrm{d} s \approx \nabla Q\left(\boldsymbol{u}_{h}\right) \cdot \boldsymbol{w} \\
& \int_{0}^{1} \nabla Q(\overline{\boldsymbol{u}}(s)) \cdot \boldsymbol{w} \mathrm{d} s \approx \frac{\nabla Q\left(\boldsymbol{u}_{h}\right)+\nabla Q\left(\boldsymbol{u}_{h^{+}}\right)}{2} \cdot \boldsymbol{w}, \\
& \int_{0}^{1} \nabla Q(\overline{\boldsymbol{u}}(s)) \cdot \boldsymbol{w} \mathrm{d} s \approx \nabla Q\left(\frac{\boldsymbol{u}_{h}}{2}+\frac{\boldsymbol{u}_{h^{+}}}{2}\right) \cdot \boldsymbol{w},
\end{aligned}
$$

where $\boldsymbol{u}_{h^{+}}$is an improved solution closer to $\boldsymbol{u}$, computed in a larger function space $\boldsymbol{V}_{h^{+}}$.
The Galerkin orthogonality (10) forces the discrete solution to the dual problem to belong to a different function space than $\boldsymbol{V}_{h}$. Using the same enlarged function space as above we thus consider the following function spaces:

$\boldsymbol{V}_{h} \subset \boldsymbol{V}_{h^{+}} \subset \boldsymbol{V}$

Carrying out the discretization we obtain the discretized dual problem; find $\boldsymbol{\varphi} \in \boldsymbol{V}_{h^{+}}$:

$a_{\mathrm{S}}^{*}\left(\boldsymbol{u}_{h^{+}}, \boldsymbol{u}_{h} ; \boldsymbol{\varphi}, \boldsymbol{v}\right)=E_{\mathrm{S}}\left(\boldsymbol{u}_{h^{+}}, \boldsymbol{u}_{h} ; \boldsymbol{v}\right) \quad \forall \boldsymbol{v} \in \boldsymbol{V}_{h^{+}}$.

Since $\boldsymbol{V}_{h^{+}} \subset \boldsymbol{V}$, the exact error representation (19) must now be replaced by an approximate one:

$\left|E\left(\boldsymbol{u}, \boldsymbol{u}_{h}\right)\right| \approx\left|\sum_{K \in T_{h}}\left(L\left(\boldsymbol{\varphi}_{h^{+}}-\pi_{h} \boldsymbol{\varphi}\right)-a\left(\boldsymbol{u}_{h}, \boldsymbol{\varphi}_{h^{+}}-\pi_{h} \boldsymbol{\varphi}\right)\right)_{K}\right|$.

A straightforward way to choose $\boldsymbol{V}_{h^{+}}$is to use one order higher finite elements than was used when discretizing the primal problem, or a refined triangulation with the same order. To reduce the overhead cost, simplified methods based on solving the dual problem in $\boldsymbol{V}_{h}$ followed by post-processing to obtain a $\varphi$ that belongs to an improved space have been proposed; see for instance [6] for developments in this direction. In this paper we will use $p$-refinement. This might seem costly, but on the other hand the computations also yields the result of the finer primal problem. This is clearly a more accurate solution which can be used instead of the coarse one. The only drawback is that we do not have as correct error representation for the fine solution as for the coarse solution.

\section{Refinement strategy}

Starting from (19) the following estimates of the error holds:

$$
\begin{aligned}
\left|E\left(\boldsymbol{u}, \boldsymbol{u}_{h}\right)\right| & =\left|\sum_{K \in T_{h}}\left(L\left(\boldsymbol{\varphi}-\pi_{h} \boldsymbol{\varphi}\right)-a\left(\boldsymbol{u}_{h}, \boldsymbol{\varphi}-\pi_{h} \boldsymbol{\varphi}\right)\right)_{K}\right|, \\
& \leqslant \sum_{K \in T_{h}}\left|\left(L\left(\boldsymbol{\varphi}-\pi_{h} \boldsymbol{\varphi}\right)-a\left(\boldsymbol{u}_{h}, \boldsymbol{\varphi}-\pi_{h} \boldsymbol{\varphi}\right)\right)\right|_{K}, \\
& \leqslant \sum_{K \in T_{h}} R_{K}\left(\boldsymbol{u}_{h}\right) \cdot W_{K}\left(\boldsymbol{\varphi}-\pi_{h} \boldsymbol{\varphi}\right) .
\end{aligned}
$$

Eq. (26) is an identity and in (27), the triangle inequality supresses cancellation between different elements. In (28) integration by parts together with the Cauchy-Schwarz inequality results in two constants $R_{K}$ and $W_{K}$ on the element level, e.g., with $a(\boldsymbol{u}, \boldsymbol{v})=\int(A \boldsymbol{u}) \cdot \boldsymbol{v}$ and $L(\boldsymbol{v})=$ $\int \boldsymbol{f} \cdot \boldsymbol{v}$, 
$R_{K}\left(\boldsymbol{u}_{h}\right)=\left\|\boldsymbol{R}_{h}\right\|_{L_{2}(K)}$,

$W_{K}\left(\boldsymbol{\varphi}-\pi_{h} \boldsymbol{\varphi}\right)=\left\|\boldsymbol{\varphi}-\pi_{h} \boldsymbol{\varphi}\right\|_{L_{2}(K)}$,

where $\boldsymbol{R}_{h} \stackrel{\text { def }}{=} \mathrm{A} \boldsymbol{u}_{\mathrm{h}}-\boldsymbol{f}$. In earlier work [10] there has been proposed the use of (26) as a stop criterion and (27) for assigning refinement indicators to the elements in $T_{h}$. This is a natural choice since elements with large contributions to (26) must be controlled. The weights used in (28) can be used to compute indicators and error bounds, but the obtained bound could be many times larger than $\left|E\left(\boldsymbol{u}, \boldsymbol{u}_{h}\right)\right|[10]$.

Furthermore, the set of element errors from (27) can be used in different ways when choosing how specific elements should be refined. We mention two common strategies and one slightly more complicated strategy together with some comments of their relative effectiveness. We define $\eta_{K}$ as the error contribution from element $K$ and the corresponding indicator as $I_{K}=$ $\left|\eta_{K}\right|$.

(1) Fixed fraction. In each refinement cycle the elements are sorted according to their indicator's magnitude $I_{K}$ and a fixed fraction are chosen to be refined. This strategy gives good control of the size of the refined mesh, but is insensitive to whether the problem has a singularity or not. Over refinements when singularities are present is common in practice.

(2) Relative fraction. In each refinement cycle the elements are sorted according to their indicator's magnitude $I_{K}$ and each element, whose indicator value is greater than or equal to a fraction of the largest value, is chosen to be refined. This strategy is more adaptive than strategy (1) and in practice, when a singularity is present, the elements surrounding the singularity are chosen to be refined. If the threshold fraction is set to low, this strategy leads to many grid levels with just a few more elements from one level to the next.

(3) Mixed refinement. Using this strategy a refinement flag between 0 and 3 is assigned to each element in the mesh. The flag indicates how many element sides that is to be divided to the next grid level. The breakpoint for flag $n \in\{1,2,3\}$ is defined by the parameter $r \in[0,1]$ and the largest indicator value $I_{K \in T_{h}}^{\max }$ in the mesh, using the following formula:

$I_{K \in T_{h}}^{\max } \cdot r^{3-n} \geqslant I_{K} \geqslant r^{4-n} \cdot I_{K \in T_{h}}^{\max }$.

This strategy balance properties from the two other strategies and is well suited for computations involving singularities. The elements that surrounds the singularity are divided into four new elements but also elements further from the singularity are refined, decreasing with the distance. Moreover, the parameter $r$ can be tuned for different approximation orders such that best possible performance is achieved.

\section{Numerical experiments-a single edge notch}

The primal problem is solved numerically on a grid hierarchy using $h$-refinement between each grid level. On each grid level we use a uniform $p$-refinement to obtain an improved solution $\boldsymbol{u}_{h^{+}} \in \boldsymbol{V}_{h^{+}}$closer to $\boldsymbol{u}$. This improved solution is used in the linearization of $E_{\mathrm{S}}\left(\boldsymbol{u}, \boldsymbol{u}_{h} ; \boldsymbol{v}\right)$, and the dual problem is then approximated in the same function space $\boldsymbol{V}_{h^{+}}$. The error representation formula is evaluated and the elements that is to be refined are chosen according to the mixed refinement strategy.

\subsection{The primal problem}

Referring to Fig. 1 we solve, with $a=0.1 \mathrm{~m}, W=0.5$ $\mathrm{m}$ and $h=\{0.5,1\} \mathrm{m}$, the equations of linear elasticity with a prescribed boundary traction $\boldsymbol{\sigma}_{0}=[0,1 \mathrm{e}+06] \mathrm{N}$. The body is in plane stress, the crack is sharp and the Lamé coefficients are $\lambda=121.154 \mathrm{e}+9$ and $\mu=$ $80.7692 \mathrm{e}+9$ corresponding to $E=210 \mathrm{e}+06 \mathrm{~Pa}$ and $v=0.3$.

$\boldsymbol{\sigma}=\lambda(\boldsymbol{\nabla} \cdot \boldsymbol{u}) \boldsymbol{I}+2 \mu \boldsymbol{\varepsilon}(\boldsymbol{u})$ in $\Omega$,

$-\nabla \cdot \sigma^{T}=\boldsymbol{f}$ in $\Omega$,

$\boldsymbol{u}=\boldsymbol{g} \quad$ on $\partial \Omega_{\mathrm{D}}$

$\boldsymbol{\sigma} \cdot \boldsymbol{n}=\boldsymbol{\sigma}_{0} \quad$ on $\partial \Omega_{\mathrm{N}}$.

Eq. (32) defines the constitutive relation between Cauchy's stress tensor and the strain field and (33) represents

$\sigma_{0}$

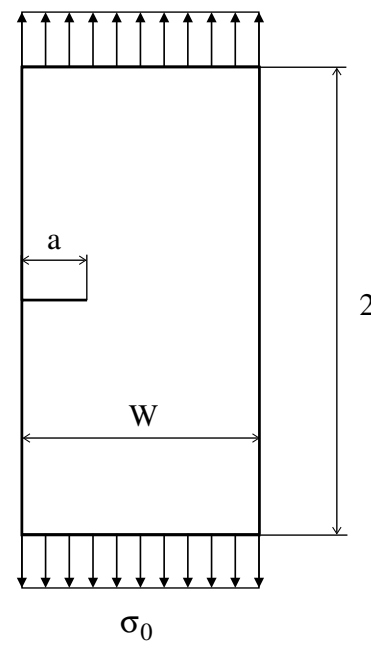

Fig. 1. The primal problem. The macroscopic crack length is $a$, and the domain width and height are $W$ and $2 h$, respectively. The boundary traction is denoted by $\sigma_{0}$. 
force equilibrium in the domain. Boundary conditions are imposed by (34) and (35) as prescribed displacements and tractions, respectively. The weak formulation of (32)-(35) is; find $\boldsymbol{u} \in \boldsymbol{V}$ :

$$
\int_{\Omega} \boldsymbol{\sigma}(u): \boldsymbol{\varepsilon}(\boldsymbol{v}) \mathrm{d} \Omega=\int_{\Omega} \boldsymbol{f} \cdot \boldsymbol{v} \mathrm{d} \Omega+\int_{\partial \Omega_{\mathrm{N}}} \boldsymbol{\sigma}_{0} \cdot \boldsymbol{v} \mathrm{d} s \quad \forall \boldsymbol{v} \in \boldsymbol{V} .
$$

In the FE approximation we choose $\boldsymbol{V}_{h}$ as the space of continuous piecewise linear, -quadratic and -cubic basis functions.

\subsection{The dual problems}

The stress field near a crack tip can be divided into three basic types, each associated with a deformation mode, see Fig. 2. Mode I is associated with tensile opening where the crack faces move directly apart. Mode II is characterized by displacements in which the crack faces slide over one another perpendicular to the leading edge of the crack. In Mode III, the crack surfaces slide parallel to the leading edge. The superposition of these three modes is often sufficient to describe a general deformation in the vicinity of the crack.

The parameters $K_{\mathrm{I}}, K_{\mathrm{II}}$ and $K_{\mathrm{III}}$ are the corresponding stress intensity factors associated with each deformation mode. Physically they represent the intensity of the linear-elastic stress distribution and from a mathematical point of view, the strengths of the $\frac{1}{\sqrt{r}}$ singularities at the crack tip. The stress intensity factors can be evaluated numerically from the displacement field or empirically from handbooks in fracture mechanics.

The relation between the stress intensity factors and the energy release rate at crack growth for a linear elastic specimen in plane stress is [11]

$J=\frac{K_{\mathrm{I}}^{2}}{E}+\frac{K_{\mathrm{II}}^{2}}{E}+\frac{(1+v) K_{\mathrm{III}}^{2}}{E}$.

The corresponding energy release rate for our model problem is thus

$J=\frac{K_{\mathrm{I}}^{2}}{E}$,

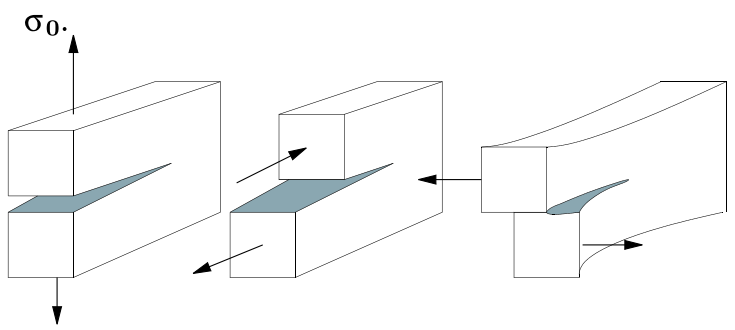

Fig. 2. Deformation modes. (1) Mode I-tensile opening, (2) Mode II - in plane shear, and (3) Mode III —out of plane shear. where the empirical expression for $K_{\mathrm{I}}$ is

$K_{\mathrm{I}}=\sqrt{\pi a} \cdot f(a, W)$,

with an accuracy better than $0.5 \%$ for any $\frac{a}{W}$ [11]. The term $f(a, W)$ is a geometry factor and it is noted that the stress intensity factor is a function of the loading conditions and geometry of the considered specimen only. The effect of $\frac{h}{W}$ is practically negligible for $\frac{h}{b} \geqslant 1.0$ [11]. The considered dimensions give the following expression for $J$ :

$J=2.7942 \pm 0.013971 \Longleftrightarrow J \in[2.7802,2.808]$.

In comparison with evaluating the stress intensity factors from the displacement field, the use of energy methods has the advantage that exact modeling of the crack tip behavior is not necessary.

In the following sections we consider three different goal quantities; crack boundary movements and two different integral formulations of the energy release rate $J$ at crack growth. The dual problems are discretized with continuous piecewise polynomials of order $p+1$, where $p$ is the order of the primal problem. The dual problems are on the form; find $\boldsymbol{\varphi} \in \boldsymbol{V}$ :

$a(\boldsymbol{\varphi}, \boldsymbol{v})=E_{\mathrm{S}}\left(\boldsymbol{u}, \boldsymbol{u}_{h} ; \boldsymbol{v}\right) \quad \forall \boldsymbol{v} \in \boldsymbol{V}$.

Remark. Since the considered equations are linear and symmetric, the secant of the semi-linear form is simply $a_{\mathrm{S}}^{*}\left(\boldsymbol{u}, \boldsymbol{u}_{h} ; \boldsymbol{\varphi}, \boldsymbol{v}\right)=a(\boldsymbol{\varphi}, \boldsymbol{v})$.

\subsubsection{Crack boundary displacements}

We choose the mean vertical displacement along the upper crack boundary $\Gamma_{a}$ as the goal quantity

$Q(\boldsymbol{u})=\frac{1}{|a|} \int_{\Gamma_{a}} \boldsymbol{u} \cdot n_{2} \mathrm{~d} s$,

where $n_{2}$ is the $x_{2}$ component of the unit normal on $\Gamma_{a}$ and $a$ is the crack length. The secant form is

$E_{\mathrm{S}}\left(\boldsymbol{u}, \boldsymbol{u}_{h} ; \boldsymbol{v}\right)=\frac{1}{a} \int_{\Gamma_{a}} \boldsymbol{v} \cdot n_{2} \mathrm{~d} s$

\subsubsection{Contour integral format}

The contour integral format of the energy release rate has been widely used as a fracture characterizing parameter. In 1968, Rice [7] showed that the value of this integral could be written as a path independent line integral for elastic materials, and thus could be evaluated at a remote contour avoiding singularity zones

$Q(\boldsymbol{u})=\int_{\Gamma}\left(W^{\mathrm{e}} \mathrm{d} x_{2}-T_{i} \frac{\partial u_{i}}{\partial x_{1}} \mathrm{~d} s\right)$. 
$W^{\mathrm{e}}$ is the elastic energy and $\boldsymbol{T} \stackrel{\text { def }}{=} \boldsymbol{\sigma} \cdot \boldsymbol{n}$ is the traction on the contour with normal $\boldsymbol{n}$. The corresponding secant form of the error measure is

$E_{\mathrm{S}}\left(\boldsymbol{u}, \boldsymbol{u}_{h} ; \boldsymbol{v}\right)=\int_{\Omega} \int_{0}^{1} \nabla Q(\overline{\boldsymbol{u}}) \cdot \boldsymbol{v} \mathrm{d} S \mathrm{~d} \Omega$,

where

$$
\begin{aligned}
{[\nabla Q(\overline{\boldsymbol{u}})]_{1}=} & \left.\left.\frac{\partial \bar{u}_{2}}{\partial x_{2}} n_{1} \lambda-n_{2} \frac{\partial \bar{u}_{1}}{\partial x_{2}} \mu+\frac{\partial \bar{u}_{2}}{\partial x_{1}}(\lambda+\mu)\right)\right) \frac{\partial(\cdot)}{\partial x_{1}} \\
& \left.\left.+\quad 2 \frac{\partial \bar{u}_{1}}{\partial x_{2}} n_{1}+\frac{\partial \bar{u}_{2}}{\partial x_{1}} n_{1}-\frac{\partial \bar{u}_{1}}{\partial x_{1}} n_{2}\right)\right) \mu \frac{\partial(\cdot)}{\partial x_{2}}
\end{aligned}
$$

$$
\begin{aligned}
{[\nabla Q(\overline{\boldsymbol{u}})]_{2}=} & \frac{\partial \bar{u}_{1}}{\partial x_{2}} n_{1} \mu-\frac{\partial \bar{u}_{1}}{\partial x_{1}} n_{2}(\lambda+\mu) \\
& \left.-\frac{\partial \bar{u}_{2}}{\partial x_{2}} n_{2}(\lambda+2 \mu)\right) \frac{\partial(\cdot)}{\partial x_{1}} \\
& \left.\left.+\frac{\partial \bar{u}_{1}}{\partial x_{1}} n_{1} \lambda+2 \frac{\partial \bar{u}_{2}}{\partial x_{2}} n_{1}-\frac{\partial \bar{u}_{2}}{\partial x_{1}} n_{2}\right)(\lambda+2 \mu)\right) \frac{\partial(\cdot)}{\partial x_{2}} .
\end{aligned}
$$

Remark. The secant form of the error measurer is linear both in $\overline{\boldsymbol{u}}$ and the test argument. It is thus possible to linearize $E_{\mathrm{S}}\left(\boldsymbol{u}, \boldsymbol{u}_{h} ; \boldsymbol{v}\right)$ exactly between the function spaces $\boldsymbol{V}_{h}$ and $\boldsymbol{V}_{h^{+}}$using the two primal solutions $\boldsymbol{u}_{h}$ and $\boldsymbol{u}_{h^{+}}$.

\subsubsection{Domain integral format}

More recent formulations of the energy release rate apply an area integration (volume in 3D) for the energy release rate. Using Gauss' theorem and a scalar weight function $q$ it is possible to rewrite the contour integral to an area integral [8]

$Q(\boldsymbol{u})=\int_{A}\left(\left[\sigma_{i j} \frac{\partial u_{j}}{\partial x_{1}}-W^{\mathrm{e}} \delta_{1 i}\right] \frac{\partial q}{\partial x_{i}}\right) \mathrm{d} A$.

The scalar function $q$ equals one at the crack tip and zero at the contour $\Gamma$ and $A$ is the integration domain i.e., the area within the contour. The secant form of $E$ is

$E_{\mathrm{S}}\left(\boldsymbol{u}, \boldsymbol{u}_{h} ; \boldsymbol{v}\right)=\int_{\Omega} \int_{0}^{1} \nabla Q(\overline{\boldsymbol{u}}) \cdot \boldsymbol{v} \mathrm{d} S \mathrm{~d} \Omega$,

where

$$
\begin{aligned}
& (\nabla Q(\overline{\boldsymbol{u}}))_{1} \\
& \left.\left.=\frac{\partial q}{\partial x_{2}} \mu \frac{\partial \bar{u}_{1}}{\partial x_{2}}+(\mu+\lambda) \frac{\partial \bar{u}_{2}}{\partial x_{1}}\right)-\lambda \frac{\partial \bar{u}_{2}}{\partial x_{2}} \frac{\partial q}{\partial x_{1}}\right) \frac{\partial(\cdot)}{\partial x_{1}} \\
& \left.\left.+\mu \frac{\partial \bar{u}_{1}}{\partial x_{1}} \frac{\partial q}{\partial x_{2}}-\mu \frac{\partial q}{\partial x_{1}} \frac{\partial \bar{u}_{2}}{\partial x_{1}}+2 \frac{\partial \bar{u}_{1}}{\partial x_{2}}\right)\right) \frac{\partial(\cdot)}{\partial x_{2}}
\end{aligned}
$$

$$
\begin{aligned}
& (\nabla Q(\overline{\boldsymbol{u}}))_{2} \\
& \left.=\left(\frac{\partial q}{\partial x_{2}} \frac{\partial \bar{u}_{1}}{\partial x_{1}}(\lambda+\mu)+\frac{\partial \bar{u}_{2}}{\partial x_{2}}(\lambda+2 \mu)\right)-\frac{\partial q}{\partial x_{1}} \frac{\partial \bar{u}_{1}}{\partial x_{2}} \mu\right) \frac{\partial(\cdot)}{\partial x_{1}} \\
& \quad+\frac{\partial q}{\partial x_{2}} \frac{\partial \bar{u}_{2}}{\partial x_{1}}(\lambda+2 \mu)-\frac{\partial q}{\partial x_{1}} \frac{\partial \bar{u}_{1}}{\partial x_{1}} \lambda \\
& \left.\left.\quad+2 \frac{\partial \bar{u}_{2}}{\partial x_{2}}(\lambda+2 \mu)\right)\right) \frac{\partial(\cdot)}{\partial x_{2}} .
\end{aligned}
$$

Remark. The secant form of the error measurer is linear both in $\overline{\boldsymbol{u}}$ and the test argument. It is thus possible to linearize $E_{\mathrm{S}}\left(\boldsymbol{u}, \boldsymbol{u}_{h} ; \boldsymbol{v}\right)$ exact between the function spaces $\boldsymbol{V}_{h}$ and $\boldsymbol{V}_{h^{+}}$using the two primal solutions $\boldsymbol{u}$ and $\boldsymbol{u}_{h^{+}}$.

\section{Numerical results}

In this section we present computational results and convergence plots for each goal quantity and approximation order. In Fig. 3 we show the initial grids and in Fig. 4 the corresponding adapted grids for the considered goal quantities.

We use two effectivity indexes $\eta_{1}$ and $\eta_{2}$ defined as

$\eta_{1}=\frac{\left|\sum_{K \in T_{h}}\left(L\left(\boldsymbol{\varphi}_{h^{+}}-\pi_{h} \boldsymbol{\varphi}\right)-a\left(\boldsymbol{u}_{h}, \boldsymbol{\varphi}_{h^{+}}-\pi_{h} \boldsymbol{\varphi}\right)\right)_{K}\right|}{Q\left(\boldsymbol{u}_{h^{+}}\right)-Q\left(\boldsymbol{u}_{h}\right)}$,

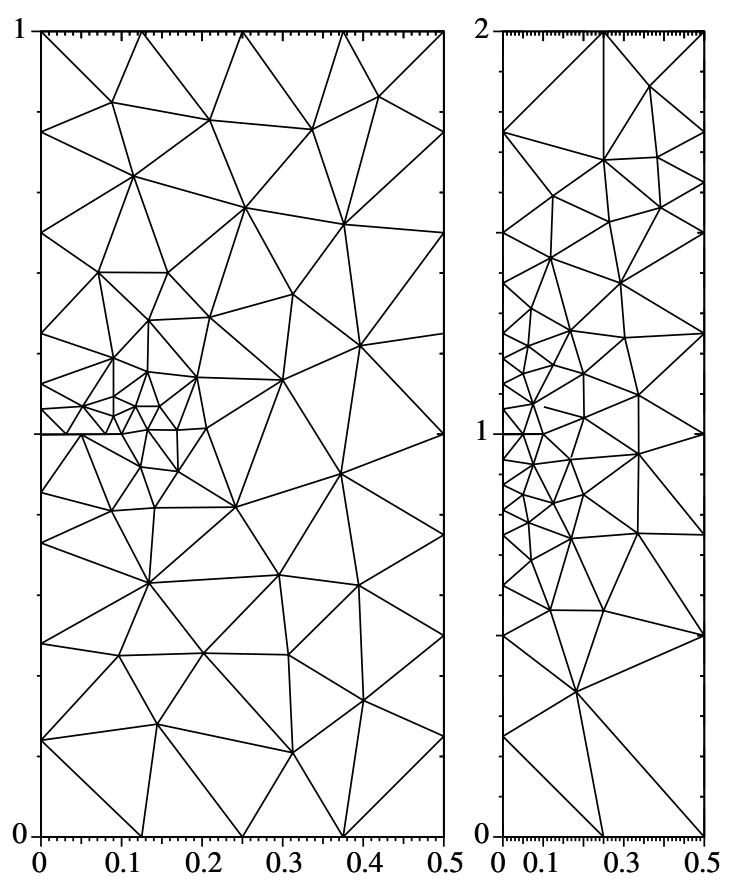

Fig. 3. The initial grids. From left to right: (1) boundary movement and (2) contour and domain integrals. 

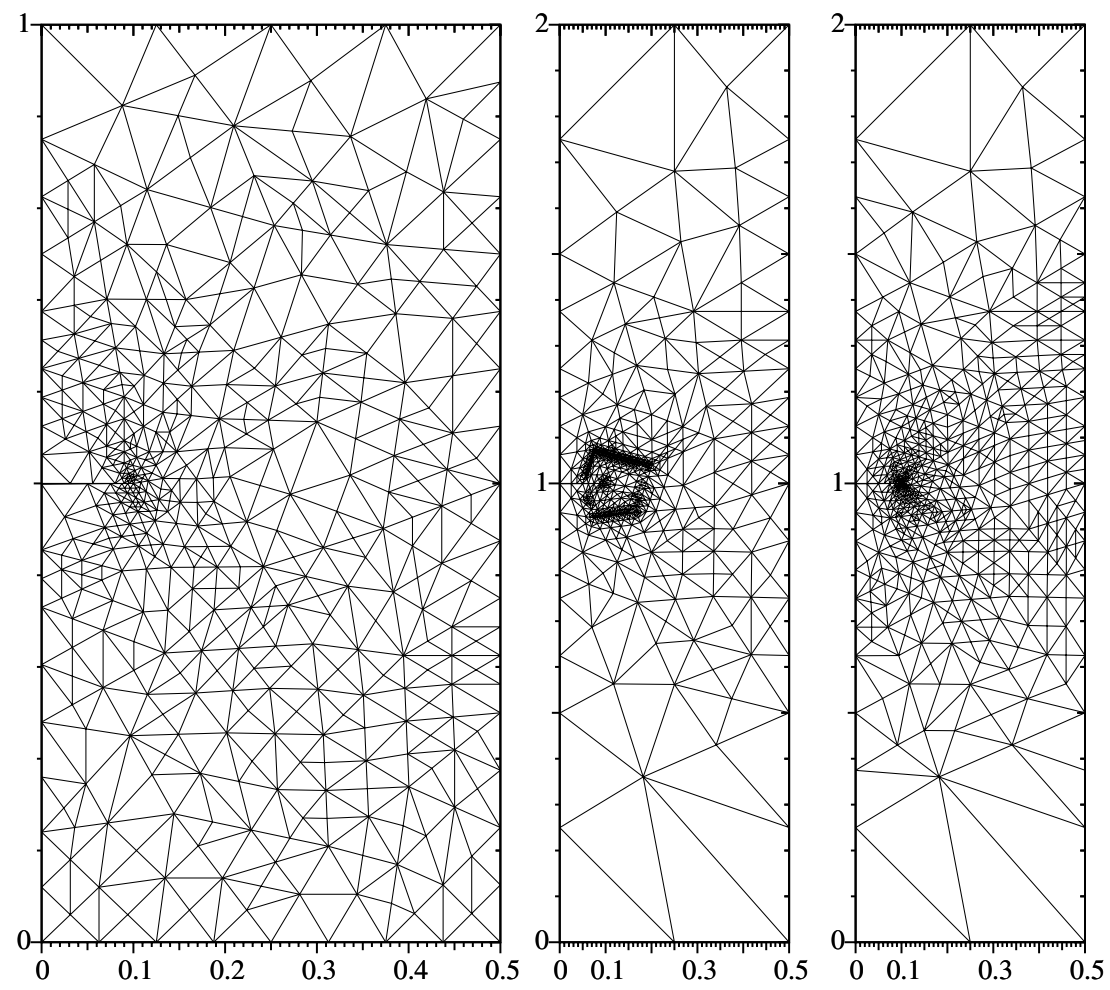

Fig. 4. Example of adapted grids for the three goal-quantities satisfying $e_{\text {rel }} \leqslant 1 \%$ for $p=1$. From left to right: (1) boundary movement, (2) contour integral and (3) domain integral.

$\eta_{2}=\frac{\left|\sum_{K \in T_{h}}\left(L\left(\boldsymbol{\varphi}_{h^{+}}-\pi_{h} \boldsymbol{\varphi}\right)-a\left(\boldsymbol{u}_{h}, \boldsymbol{\varphi}_{h^{+}}-\pi_{h} \boldsymbol{\varphi}\right)\right)_{K}\right|}{Q(\boldsymbol{u})-Q\left(\boldsymbol{u}_{h}\right)}$,

i.e., the fraction between the error representation formula and the two primal problems is $\eta_{1}$ and the fraction between the error representation formula and the difference between the converged solution and the current FE solution is $\eta_{2}$. In order to present graphs of convergence rates, we also define the average size of the elements in the grid as

$h_{a}=\frac{m(\Omega)}{\sqrt{N_{\mathrm{el}}}}$,

where $N_{\text {el }}$ is the number of elements in the current mesh and $m(\Omega)$ is the area of the considered domain.

\subsection{Boundary movements}

The exact goal quantity, $Q(\boldsymbol{u})$, was approximated by a reference solution (an adaptive computation), which was considered accurate when eight decimal points did not change from one grid level to the next.
$Q(\boldsymbol{u})=3.87362666 \mathrm{e}-07$.

Convergence and effectivity index plots can be found in Figs. 5-7. Note that the convergence rate for $p=2$ was highest for the primal problem of order $p+1$ although the error representation formula is evaluated for the primal problem of order $p$. In Fig. 7 it is shown that $\eta_{1} \approx 1$ in each iteration and $\eta_{2} \rightarrow 1$ when $h \rightarrow 0$.

\subsection{Contour integral}

$E_{\mathrm{S}}\left(\boldsymbol{u}, \boldsymbol{u}_{h}, \boldsymbol{v}\right)$ was linearized using the midpoint rule (22) and two FEM solutions $\boldsymbol{u}_{h}$ and $\boldsymbol{u}_{h^{+}}$obtained from the primal problem. This linearization is exact when $\boldsymbol{u}_{h^{+}} \rightarrow \boldsymbol{u}$ since the gradient of the goal quantity is linear in $\boldsymbol{u}$. The exact goal quantity was approximated by a reference solution (an adaptive computation), which was considered accurate if more than 245,000 degrees of freedom were used.

$Q(\boldsymbol{u})=2.7933302$.

Convergence and effectivity index plots can be found in Figs. 8-10. Note that the highest convergence rate was obtained with $p=2$ for the primal problem of order 


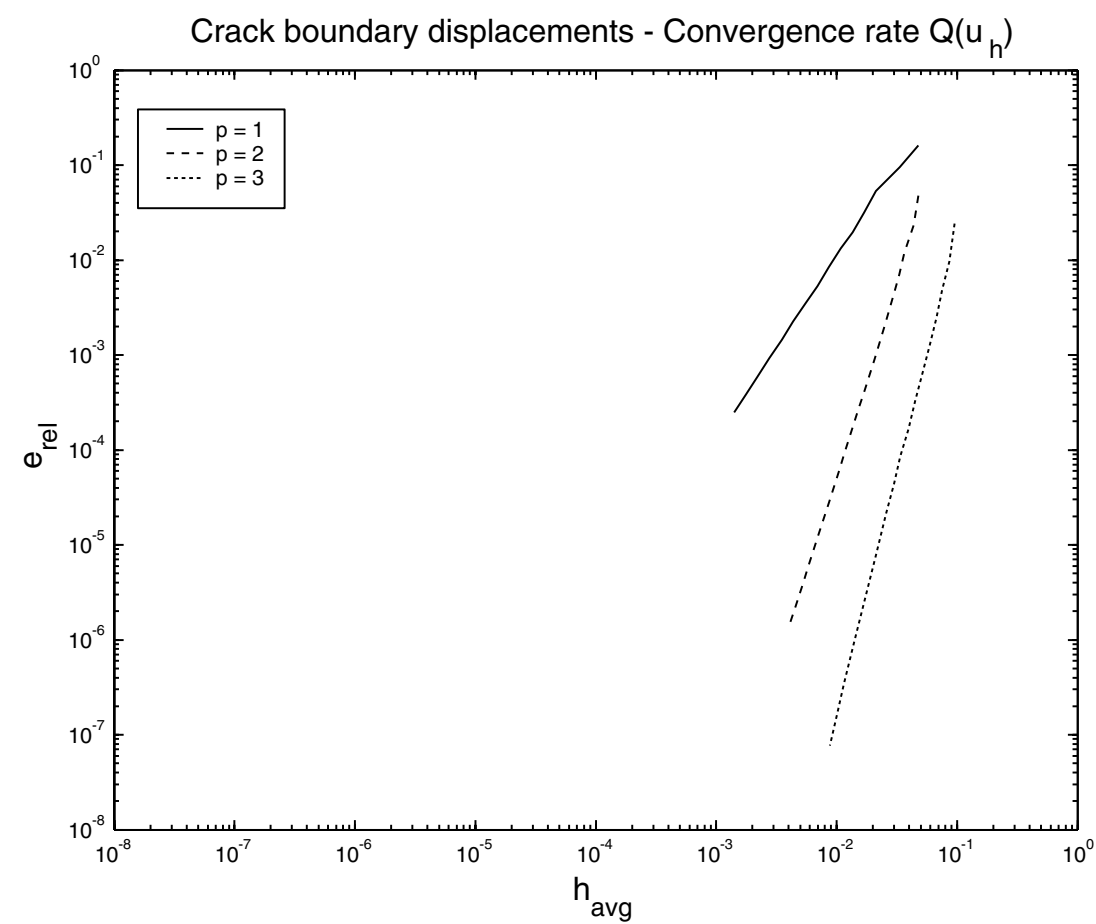

Fig. 5. Convergence rates for boundary movement. The plot shows the convergence rate for the primal problem of order $p$.

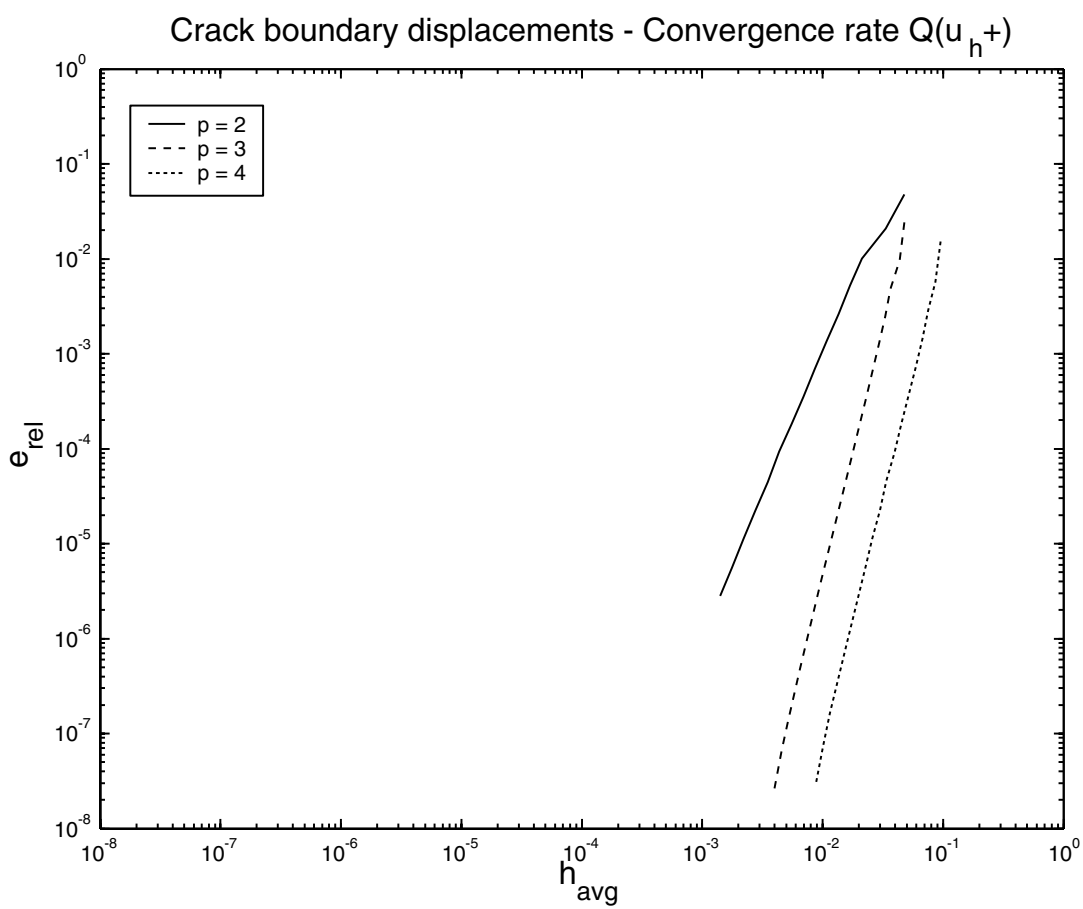

Fig. 6. Convergence rates for boundary movement. The plot shows the convergence rate for the primal problem of order $p+1$. 


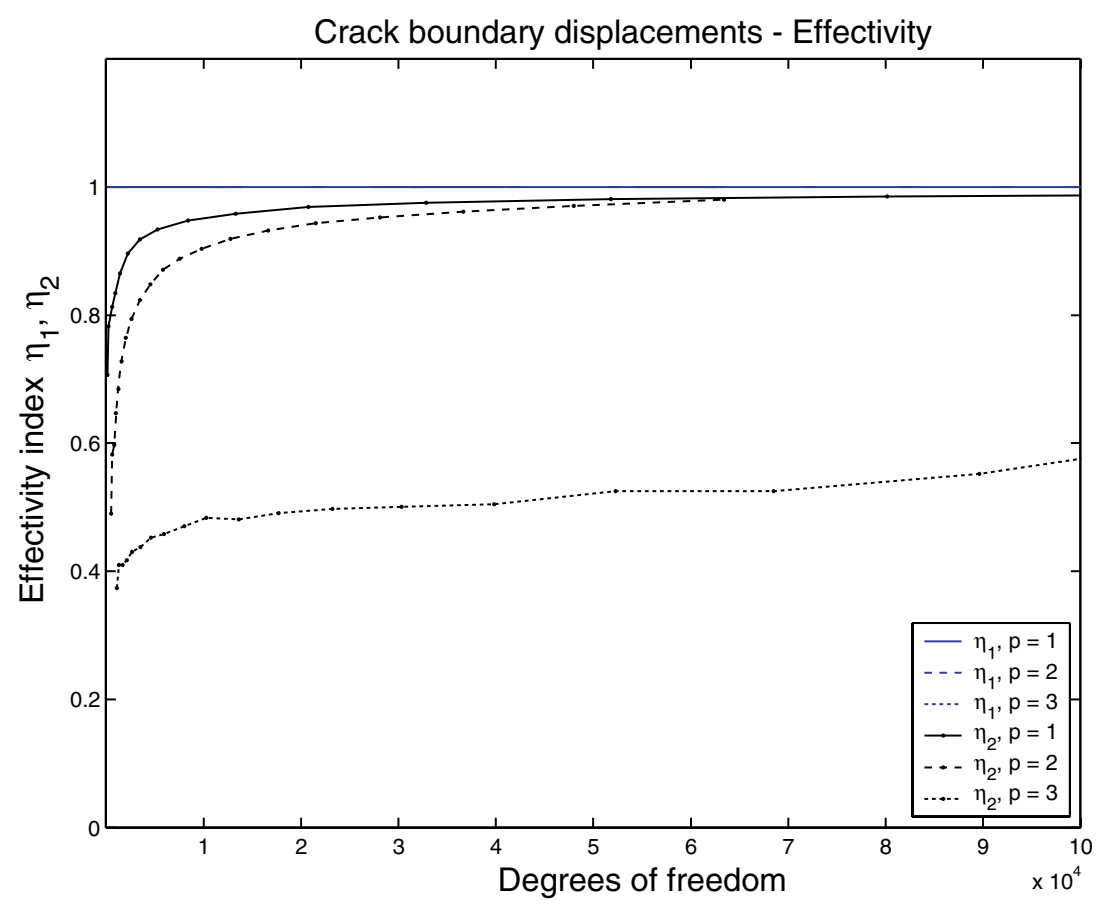

Fig. 7. Effectivity for boundary movement.

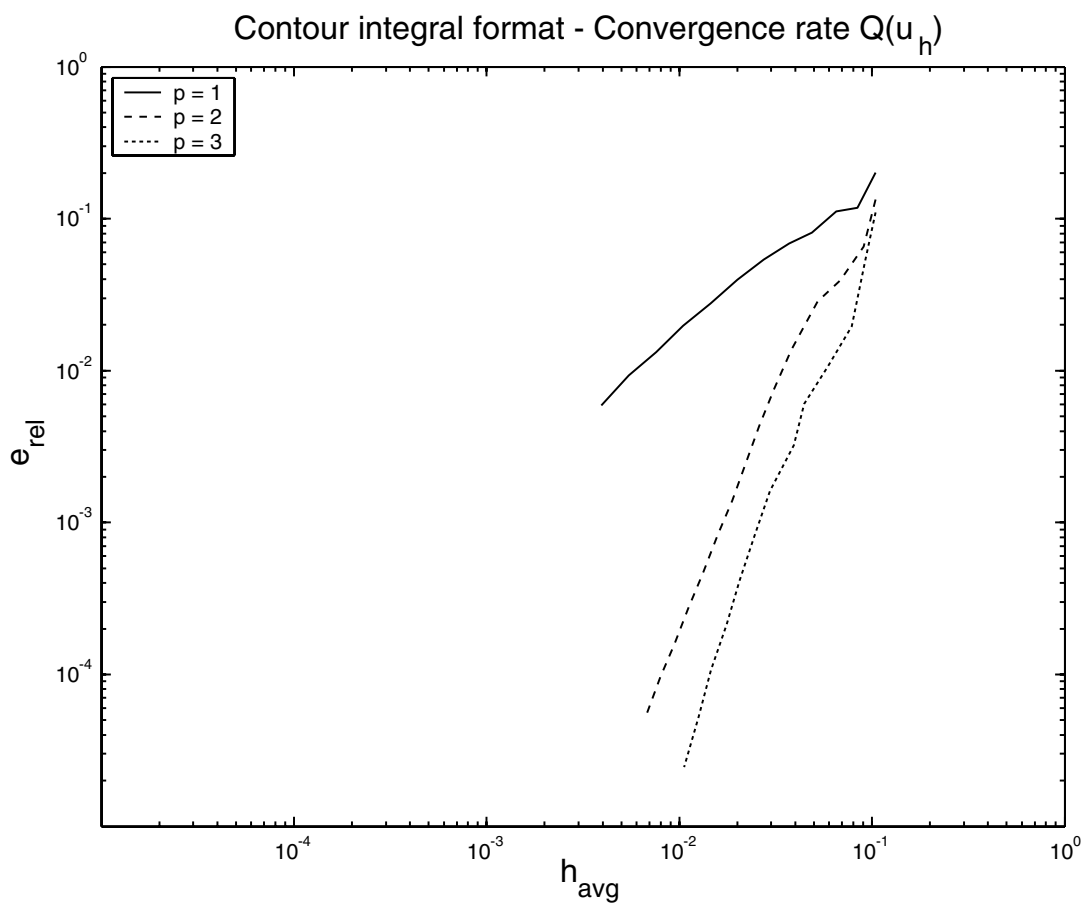

Fig. 8. Convergence rates for the contour integral formulation of $J$. The plot shows the convergence rate for the primal problem of order $p$. 


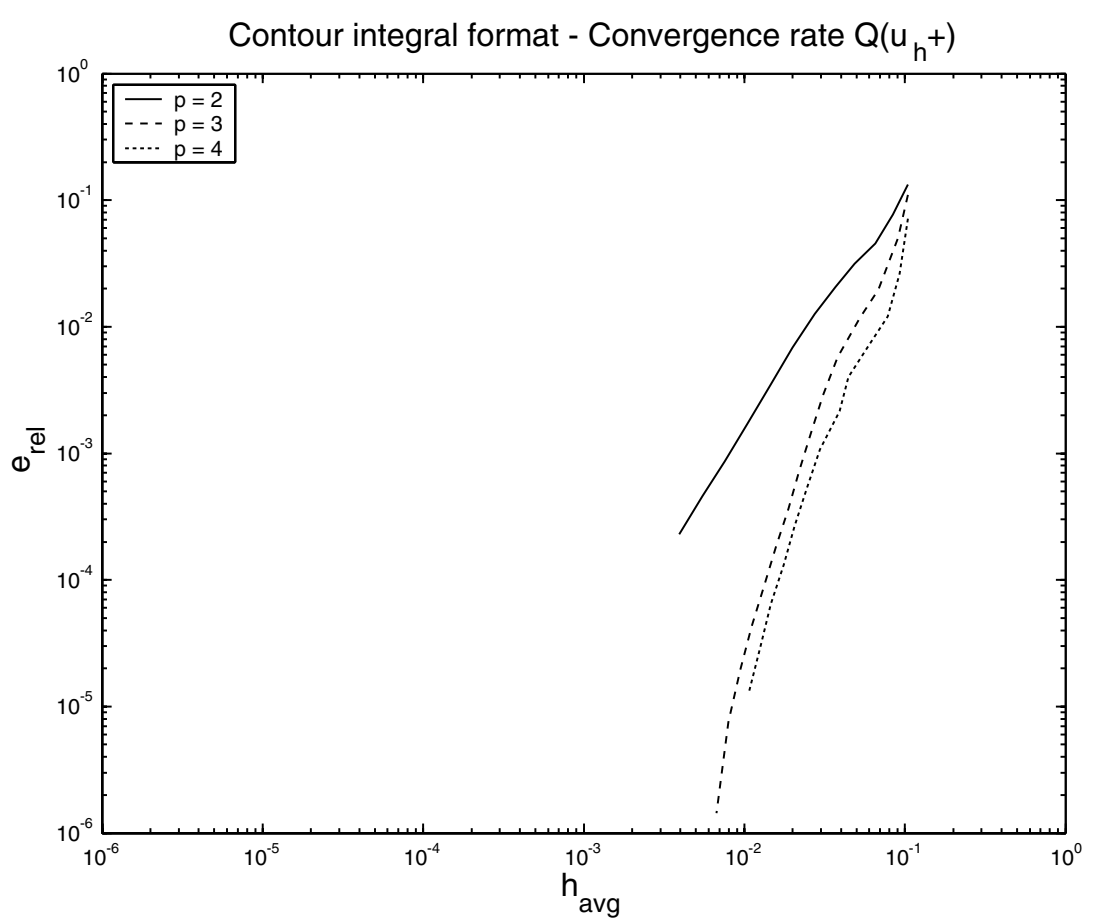

Fig. 9. Convergence rates for the contour integral formulation of $J$. The plot shows the convergence rate for the primal problem of order $p+1$.

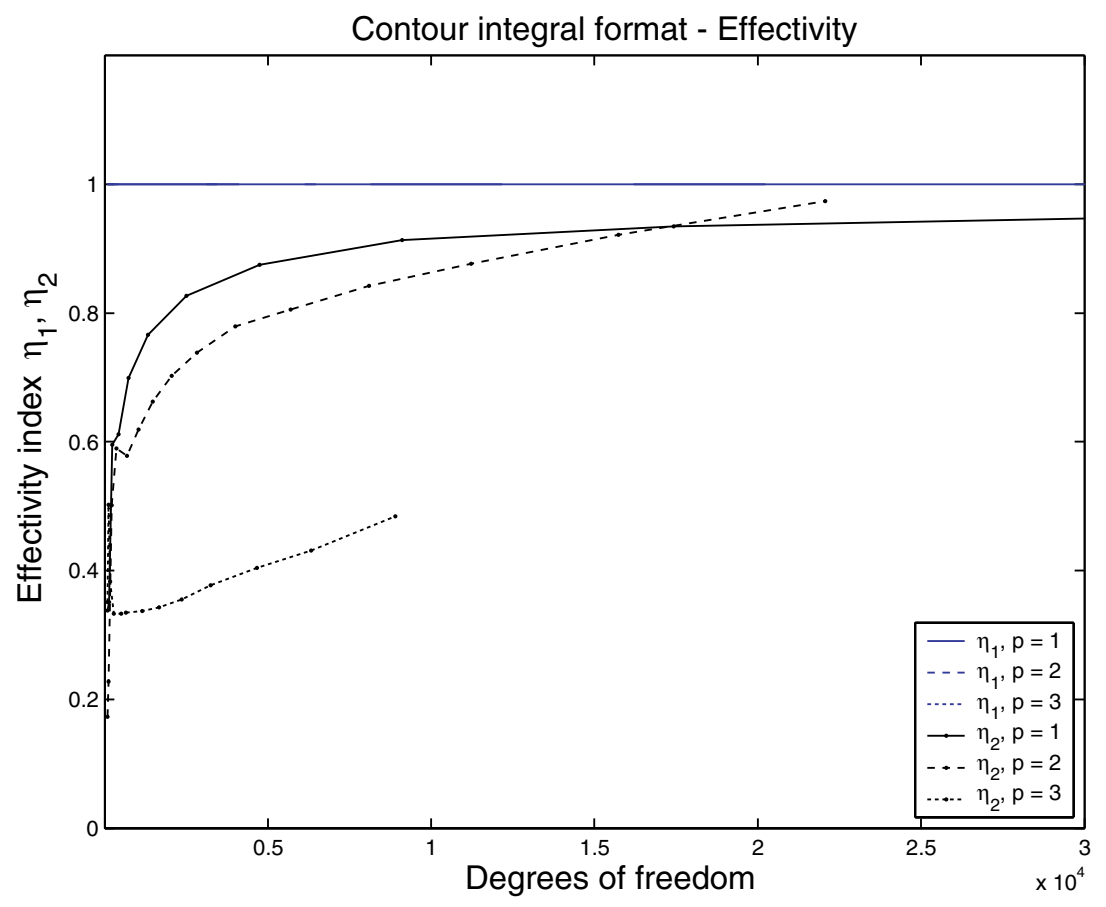

Fig. 10. Effectivity for contour integral formulation. 


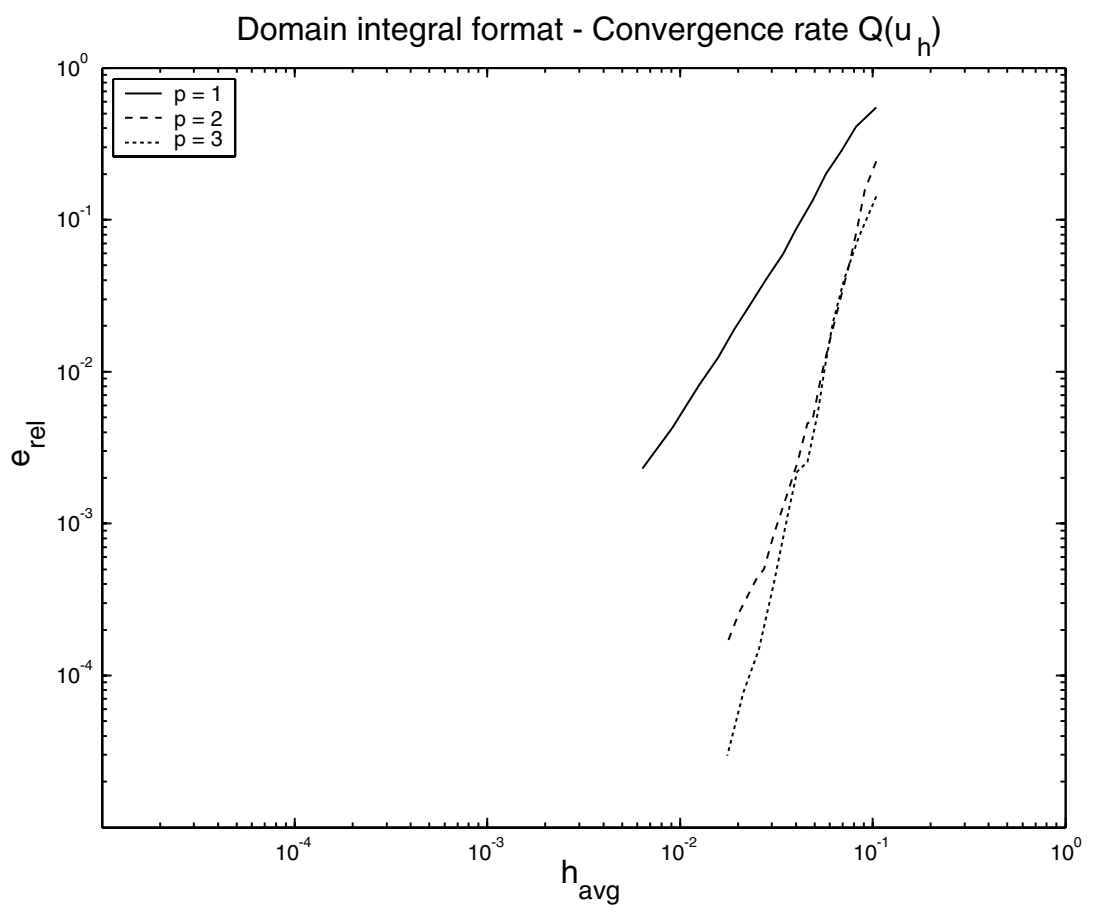

Fig. 11. Convergence rates for the domain integral formulation for $J$. The plot shows the convergence rate for the primal problem of order $p$.

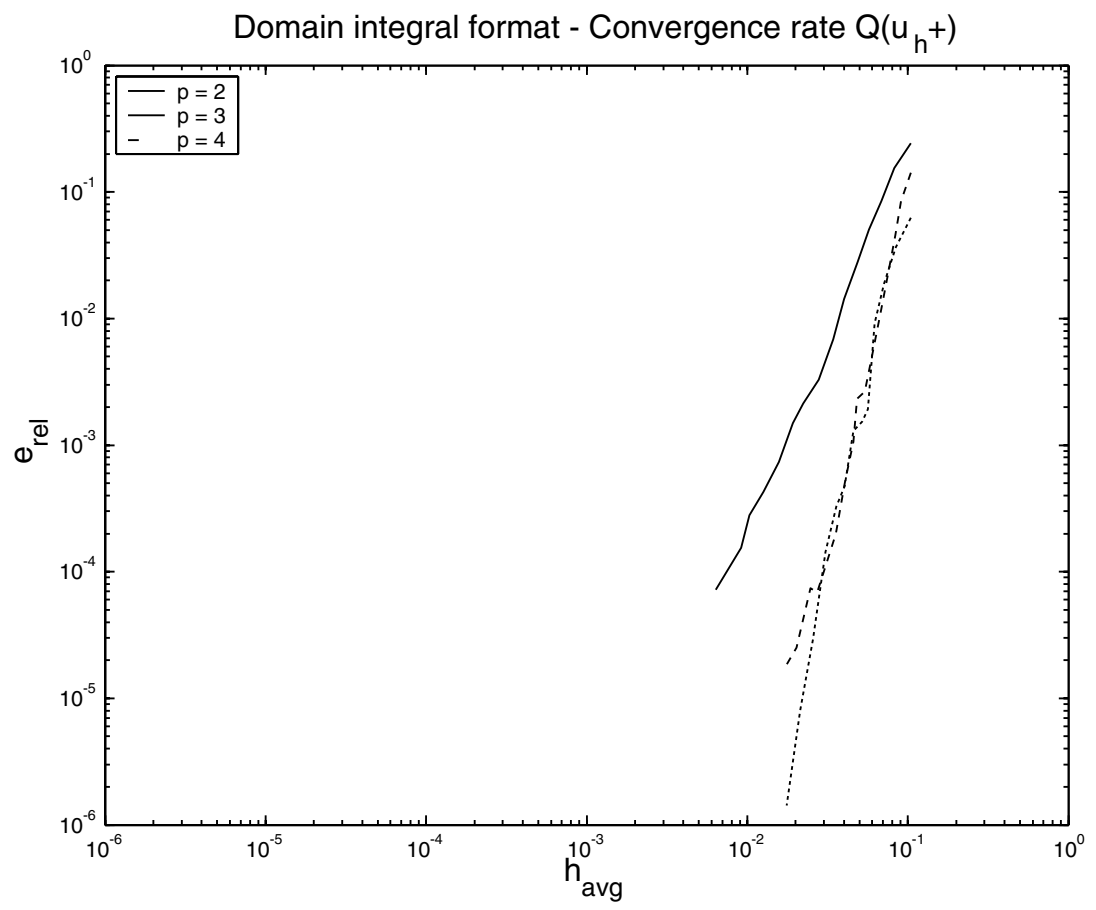

Fig. 12. Convergence rates for the domain integral formulation for $J$. The plot shows the convergence rate for the primal problem of order $p+1$. 


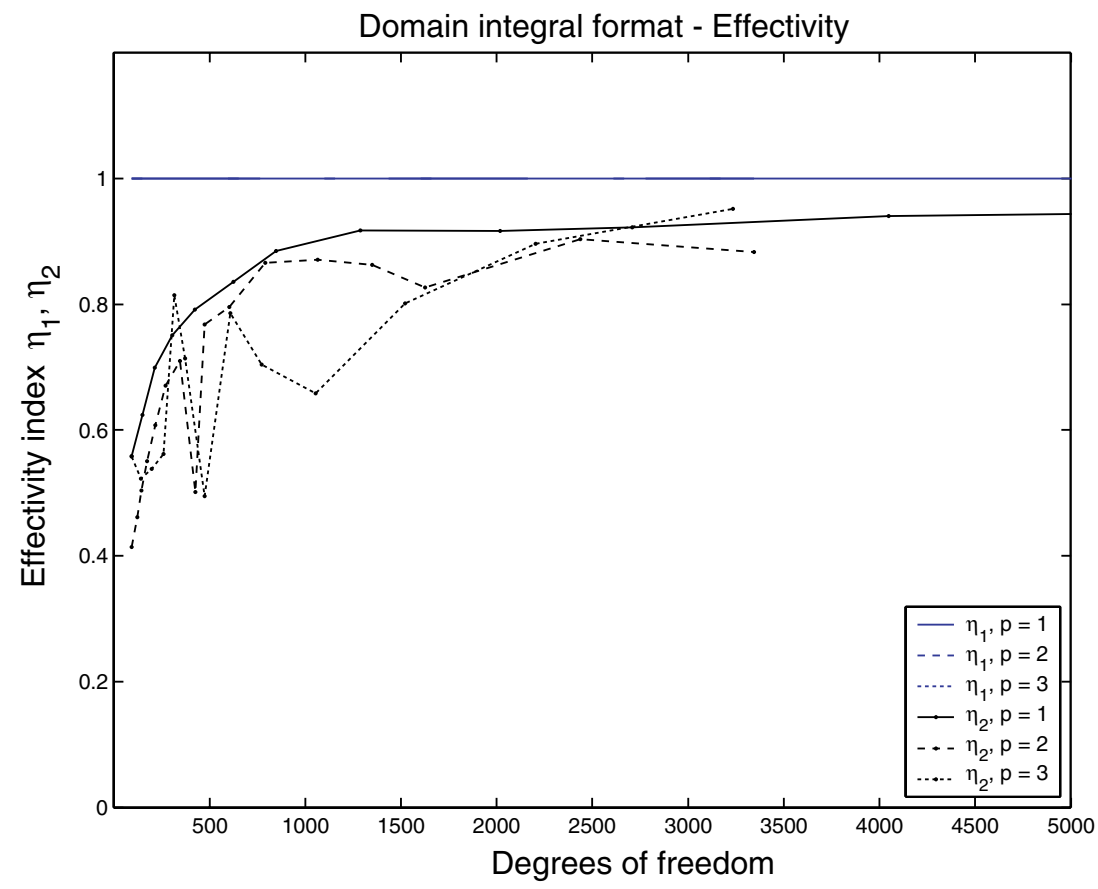

Fig. 13. Effectivity for domain integral formulation.

$p+1$. In Fig. 10 it is shown that $\eta_{1} \approx 1$ in each iteration and $\eta_{2} \rightarrow 1$ when $h \rightarrow 0$.

\subsection{Domain integral}

$E_{\mathrm{S}}\left(\boldsymbol{u}, \boldsymbol{u}_{h}, \boldsymbol{v}\right)$ was linearized using the midpoint rule (22) and two FEM solutions $\boldsymbol{u}_{h}$ and $\boldsymbol{u}_{h^{+}}$obtained from the primal problem. This linearization is exact when $\boldsymbol{u}_{h^{+}} \rightarrow \boldsymbol{u}$ since the gradient of the goal quantity is linear in $\boldsymbol{u}$. The exact goal quantity was approximated by a reference solution, which was considered accurate if more than 245,000 degrees of freedom were used.

$Q(\boldsymbol{u})=2.7953116$.

Convergence and effectivity index plots can be found in Figs. 11-13. In Fig. 13 it is shown that $\eta_{1} \approx 1$ in each iteration and $\eta_{2} \rightarrow 1$ when $h \rightarrow 0$.

\section{Concluding remarks}

In the plots we have seen that the convergence rates for elements with higher approximation order $(p=3,4)$ are influenced by the regularity of the solution (singularity at the crack tip), and does not always realize the theoretical predictions. Higher convergence rates might be obtained with the development of anisotropic mesh refinement algorithms that are more adaptive, i.e., does not take into account the element aspect ratio. See for instance [12] for recent developments of an anisotropic mesh refinement strategy based on solving a dual problem.

We have also shown that the ratio between the error representation formula and the difference between the two primal problems $\left(\eta_{1}\right)$ is close to one in each iteration. Therefore, we can say that the error estimate is in a sense trivial and that the error representation formula is more a tool to put high quality indicators on the elements for the refinement to the next grid level. This is the case for many engineering quantities such as stresses, strains and strain energy, which are at most quadratic in the solution. Thus, solve two primal problems in $\boldsymbol{V}_{h}$ and $\boldsymbol{V}_{h^{+}}$and solve the dual problem in $\boldsymbol{V}_{h^{+}}$, then

$$
\begin{aligned}
& \frac{\left|\sum_{K \in T_{h}}\left(L\left(\boldsymbol{\varphi}_{h^{+}}-\pi_{h} \boldsymbol{\varphi}\right)-a\left(\boldsymbol{u}_{h}, \boldsymbol{\varphi}_{h^{+}}-\pi_{h} \boldsymbol{\varphi}\right)\right)_{K}\right|}{Q\left(\boldsymbol{u}_{h^{+}}\right)-Q\left(\boldsymbol{u}_{h}\right)} \\
& \quad \approx 1.0,
\end{aligned}
$$

should hold. Satisfaction of (58) may be used as a check of the correctness of the FE formulation and the FE code.

The overhead cost of the evaluation of two primal problems is high, but on the other hand one can use the result of the finer primal problem. This solution is clearly more accurate, but on the other hand the error representation is less correct than for the coarse solu- 
tion. In the numerical examples we have seen that the best overall results have been obtained with $p=2$ for the primal and $p=3$ for the dual problem.

\section{References}

[1] Eriksson K, Estep D, Hansbo P, Johnson C. Computational differential equations. Cambridge University Press; 1996.

[2] Eriksson K, Estep D, Hansbo P, Johnson C. Introduction to adaptive methods for differential equations. Acta Numer 1995:105-58.

[3] Johnson C, Hansbo P. Adaptive finite element methods in computational mechanics. Comp Meth Appl Mech Eng 1992;(101):143-81.

[4] Becker R, Rannacher R. A feed-back approach to error control in finite element methods: basic analysis and examples. East-West J Numer Math 1996;(4):237-64.

[5] Rannacher R, Suttmeier F-T. A feed-back approach to error control in finite element methods: application to linear elasticity. Comput Mech 1997;(19):434-46.
[6] Larsson F. Goal-oriented error measures and adaptive finite element procedures in solid mechanics. lic. thesis, Department of Solid Mechanics, Chalmers University of Technology, 2001.

[7] Rice JR. A path independent integral and the approximate analysis of strain concentration by notches and cracks. J Appl Mech 1968;35:379-86.

[8] Li FZ, Shih CF, Needleman A. A comparison of methods for calculating energy release rates. Eng Fract Mech 1985;21:405-21.

[9] Shih CF, Moran B, Nakamura T. Energy release rate along a three-dimensional crack front in a thermally stressed body. Int J Fract 1986;30:79-102.

[10] Larson M, Niklasson J. A posteriori error estimation of functionals in elliptic problems: Experiments. Technical report, Chalmers Finite Element Center, 2001. PREPRINT 2001-04.

[11] Tada H, Paris PC, Irwin GR. The stress analysis of cracks handbook. 3rd ed. ASME press; 2000.

[12] Larsson F. A simple anisotropic mesh-refinement strategy for triangular elements in 2D. PhD thesis, Department of Applied Mechanics, Chalmers University of Technology, submitted for publication. 